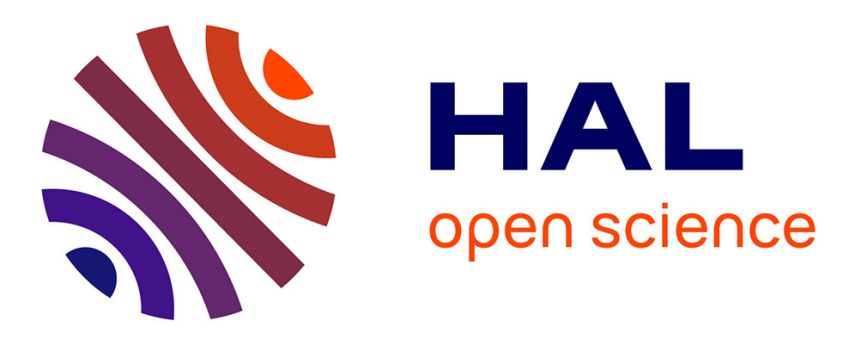

\title{
LMI-based discrete-time nonlinear state observer for an anaerobic digestion model
}

Khadidja Chaib Draa, Holger Voos, Marouane Alma, Ali Zemouche, Mohamed Darouach

\section{- To cite this version:}

Khadidja Chaib Draa, Holger Voos, Marouane Alma, Ali Zemouche, Mohamed Darouach. LMI-based discrete-time nonlinear state observer for an anaerobic digestion model. 6th International Conference on Systems and Control, ICSC 2017, May 2017, Batna, Algeria. 10.1109/icosc.2017.7958714 . hal01567354

\section{HAL Id: hal-01567354 \\ https://hal.science/hal-01567354}

Submitted on 12 Jan 2018

HAL is a multi-disciplinary open access archive for the deposit and dissemination of scientific research documents, whether they are published or not. The documents may come from teaching and research institutions in France or abroad, or from public or private research centers.
L'archive ouverte pluridisciplinaire HAL, est destinée au dépôt et à la diffusion de documents scientifiques de niveau recherche, publiés ou non, émanant des établissements d'enseignement et de recherche français ou étrangers, des laboratoires publics ou privés. 


\title{
LMI-Based Discrete-Time Nonlinear State Observer for an Anaerobic Digestion Model
}

\author{
K. Chaib Draa, H. Voos, M. Alma, A. Zemouche and M. Darouach
}

\begin{abstract}
This paper deals with the design of a discrete time nonlinear observer for an anaerobic digestion process. The designed observer is devoted to a general class of systems, precisely linear systems, LPV systems with known and bounded parameters, and nonlinear Lipschitz systems. In order to ensure stability of the estimation error, a new LMI condition is proposed. In this LMI, additional decision variables are included to enhance its feasibility. Indeed, this was possible due to the use of a suitable reformulation of the Young's inequality. Numerical simulations using the investigated two-step anaerobic digestion model show the effectiveness of the proposed LMI methodology.
\end{abstract}

Index Terms - Anaerobic digestion, LMI approach, Observer Design, Nonlinear systems.

\section{INTRODUCTION}

A fundamental problem in Anaerobic Digestion (AD) monitoring and supervision is how to access the process state variables. Indeed, some key variables are very important to measure for controlling the process and prevent its disruption and failure. However, in practice some of those key variables (bacteria concentration for instance) may not be measurable due the lack of appropriate measurement devices, or the high cost of these devices. An alternative solution for such a problem is the design of software sensors (observers) to estimate the unmeasurable state variables. Nevertheless, designing an observer for AD model is not an easy task due to the complexity of its dynamics and the absence of key measurements.

Among the designed observers for AD model, we can cite the asymptotic observer [1] which is quite simple and does not require the knowledge of some specific nonlinear functions. However, such an observer is very sensitive to model uncertainties and its convergence rate depends on the operating conditions. Therefore, it has been extended to interval observers [2] which have the advantage of using reliable measurements, which are nonlinear functions of the state vector. The interval observers estimate the interval where the state is lying when the system has some uncertainties. However, generally the rate of convergence is partially tunable and it is not easy to exploit the estimated intervals for control. Of course observers with tunable rate of convergence have also been proposed in the literature. We find a lot

Khadidja CHAIB DRAA and Holger VOOS are with the Interdisciplinary Centre for Security, Reliability and Trust (SnT)- Université du Luxembourg. khadidja.chaibdraa@uni.lu

Marouane ALMA and Mohamed DAROUACH are with the CRAN-CNRS, Université de Lorraine, IUT de Longwy.

Ali ZEMOUCHE is with University of Lorraine, CRAN CNRS UMR 7039, 54400 Cosnes et Romain, France, and with EPI INRIA DISCO, Laboratoire des Signaux et SystẦ̀mes, CNRS-Supélec, 91192 Gif-sur-Yvette, France. of papers dealing with the Kalman filtering which shows suitable results in different chemical applications. However, convergence of the estimation arror to zero is not guarantied. The high gain observer [3] also converges fast enough to the model state variables, however its synthesis is complex and is very sensitive to noise. Moreover, in the majority of papers dealing with the design of observers for AD models, the authors assume that measurements are available on line. Whereas, this is not true in real applications. Hence, the observer operates in discrete time and is driven by discrete time measurements (sampled data) [4]. Consequently, we design in the current paper a suitable discrete time nonlinear observer for an $\mathrm{AD}$ model. The designed observer is simple to implement and its rate of convergence is tunable. Moreover, we provide a novel algorithm to ensure the stability of the estimation error using LMI techniques. Indeed, we give a new and less conservative LMI to find the observer gains. This was possible due to the use of a suitable reformulation of the Young's inequality [5]. Actually, we include additional decision variables in the new LMI to enhance its feasibility.

The rest of the paper is organized as follows. Section II, is devoted to the modelling of a controlled AD process. Section III, discusses the observer design and its stability. Section IV, is dedicated to the simulation results, while Section V concludes the paper.

Notations: The following notations will be used throughout this paper

- $(\star)$ is used for the blocks induced by symmetry;

- $A^{T}$ represents the transposed matrix of $A$;

- $\mathbb{I}_{r}$ represents the identity matrix of dimension $r$;

- for a square matrix $S, S>0(S<0)$ means that this matrix is positive definite (negative definite);

- the set $C o(x, y)=\{\lambda x+(1-\lambda) y, 0 \leq \lambda \leq 1\}$ is the convex hull of $\{x, y\}$;

- $e_{s}(i)=(\underbrace{0, \ldots, 0, \overbrace{1}^{i \text { th }}, 0, \ldots, 0}_{s \text { components }})^{T} \in \mathbb{R}^{s}, s \geq 1$ is a vector of the canonical basis of $\mathbb{R}^{s}$;

- $\mathbb{R}_{+}^{n}$ for the positive orthant of dimension $n$.

\section{Process Modeling}

AD modelling has been widely investigated in the literature, plenty models have been proposed to reproduce as close as possible its behaviour. The difference between the proposed models is linked to the application objectives and to the considered number of bacteria [6]. Nowadays, the two step (acidogenesis-methanogenesis) model (AM2) developed in [7] has become a reference in modelling of 
two step reaction processes. Therefore, we have adopted it and adapted it for further research objectives as depicted in Figure 1.

The structure of the reference model (AM2) does not change, except that the authors have introduced $S_{2 a d}$ and $Z_{a d}$ as added control inputs to provide more freedom degree when the purpose is to control the biogas quality. The other control inputs are $u_{1}=\frac{F_{1 i n}}{v}$ and $u_{2}=\frac{F_{2 i n}}{v}$ and since the digester volume $(v)$ is constant, then $u_{\text {out }}=u_{1}+u_{2}$. Thus, the mass balance model of the system depicted in Figure 1 is given by equations (1a-1f), (2), (3), (4) and (5), where $x_{1}$ is the concentration of the organic matter to be digested, $x_{2}$ the concentration of acidogenic bacteria the responsible for the organic matter degradation. The volatile fatty acids concentration $x_{3}$ is supposed to be pure acetate, $x_{4}$ is the concentration of methanogenic bacteria, $x_{5}$ represents the inorganic carbon concentration and $x_{6}$ the alkalinity concentration (it is the sum of bicarbonate (bic) and acid concentrations, as given by equations (4)). The rest of the parameters used in the model are defined Table I.

$$
\begin{aligned}
\dot{x}_{1}= & -k_{1} \mu_{1}\left(x_{1}\right) x_{2}+u_{1} S_{1 \text { in }}-u_{\text {out }} x_{1} \\
\dot{x}_{2}= & \mu_{1}\left(x_{1}\right) x_{2}-u_{\text {out }} x_{2} \\
\dot{x}_{3}= & k_{2} \mu_{1}\left(x_{1}\right) x_{2}-k_{3} \mu_{2}\left(x_{3}\right) x_{4}-u_{\text {out }} x_{3}+ \\
& u_{1}\left(S_{2 \text { in }}+S_{2 a d}\right) \\
\dot{x}_{4}= & \mu_{2}\left(x_{3}\right) x_{4}-u_{\text {out }} x_{4} \\
\dot{x}_{5}= & k_{4} \mu_{1}\left(x_{1}\right) x_{2}+k_{5} \mu_{2}\left(x_{3}\right) x_{4}+u_{1} C_{\text {in }}- \\
& u_{\text {out }} x_{5}-q_{c}(x) \\
\dot{x}_{6}= & u_{1} Z_{\text {in }}+u_{2} Z_{\text {ad }}-u_{\text {out }} x_{6}
\end{aligned}
$$

with

$$
\left\{\begin{array}{l}
\mu_{1}\left(x_{1}\right)=\bar{\mu}_{1} \frac{x_{1}}{x_{1}+k_{s_{1}}} \\
\mu_{2}\left(x_{3}\right)=\bar{\mu}_{2} \frac{x_{3}}{x_{3}+k_{s 2}+\frac{x_{3}^{2}}{k_{i 2}}}
\end{array}\right.
$$

and the gaseous flow rates, for methane $\left(q_{m}\right)$ and carbon dioxide $\left(q_{c}\right)$, are expressed by

$$
\left\{\begin{array}{l}
q_{m}(x)=k_{6} \mu_{2}\left(x_{3}\right) x_{4} \\
c o_{2}=x_{5}+x_{3}-x_{6} \\
q_{c}(x)=k_{L a}\left[c o_{2}-K_{H} P_{C}(x)\right] \\
P_{C}(x)=\frac{\phi-\sqrt{\phi^{2}-4 K_{H} P_{T} c o_{2}}}{2 K_{H}} \\
\phi=c O_{2}+K_{H} P_{T}+\frac{k_{6}}{k_{L a} \mu_{2}\left(x_{3}\right) x_{4}}
\end{array}\right.
$$

We also have

$$
\left\{\begin{array}{l}
\text { bic }=x_{6}-x_{3} \\
c o_{2}=x_{5}-b i c \\
k_{b}=\frac{\left[H^{+}\right] b i c}{c o_{2}} \\
p H=-\log _{10}\left(k_{b} \frac{c o_{2}}{b i c}\right)
\end{array}\right.
$$

We divide the system output into linear $\left(y_{1}\right)$ and nonlinear $\left(y_{2}\right)$ outputs

$$
\left\{\begin{array}{l}
y_{1}=\left[x_{1}, x_{3}, x_{6}\right]^{T} \\
y_{2}=q_{c}(x)
\end{array}\right.
$$

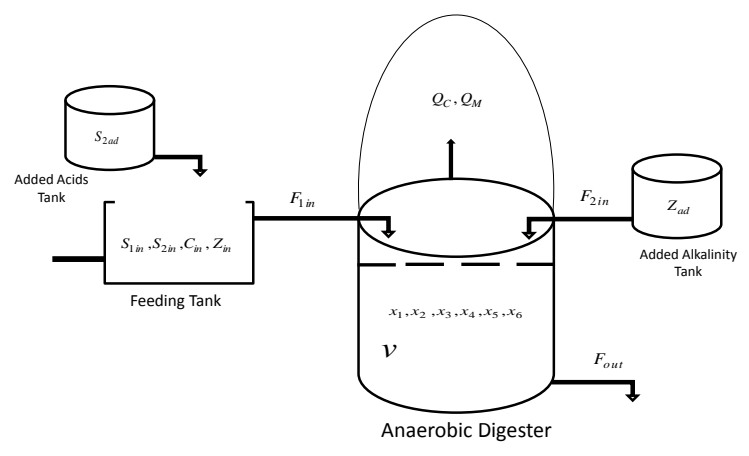

Fig. 1. Controlled Anaerobic Digestion Process.

\section{OBSERVER DESIGN AND CONVERGENCE ANALYSIS}

\section{A. System description and assumptions}

To render the proposed state observer design method general and applicable for other nonlinear models, and for clarity of the presentation, we will present the results in a general way.

Motivated by the model of anaerobic digestion (1a-1f), we will investigate the general class of discrete-time systems described by the following equations

$$
\left\{\begin{array}{l}
x_{k+1}=A\left(\rho_{k}\right) x_{k}+B \gamma\left(x_{k}\right)+g\left(y_{k}, u_{k}\right) \\
y_{k}=C x_{k}
\end{array}\right.
$$

where $x_{k} \in \mathbb{R}^{n}$ is the state vector, $y_{k} \in \mathbb{R}^{p}$ is the output measurement, $u_{k} \in \mathbb{R}^{q}$ is an input vector and $\rho_{k} \in \mathbb{R}^{s}$ is an $\mathcal{L}_{\infty}$ bounded and known parameter. The affine matrix $A\left(\rho_{k}\right)$ is expressed under the form

$$
A\left(\rho_{k}\right)=A_{0}+\sum_{j=1}^{s} \rho_{k}^{j} A_{j}
$$

with

$$
\rho_{\min }^{j} \leq \rho_{k}^{j} \leq \rho_{\max }^{j}
$$

which means that the parameter $\rho_{k}$ belongs to a bounded convex set for which the set of $2^{s}$ vertices can be defined by

$$
\mathbb{V}_{\rho}=\left\{\varrho \in \mathbb{R}^{s}: \varrho^{j} \in\left\{\rho_{\min }^{j}, \rho_{\max }^{j}\right\}\right\}
$$

The matrices $A_{i} \in \mathbb{R}^{n \times n}, B \in \mathbb{R}^{n \times m}, C \in \mathbb{R}^{p \times n}$ are constant. The nonlinear function $\gamma: \mathbb{R}^{n} \longrightarrow \mathbb{R}^{m}$ is assumed 
TABLE I

Model Parameters [7]

\begin{tabular}{|c|c|c|c|}
\hline Acronyms & Definition & Units & Value \\
\hline$k_{1}$ & Yield for substrate $\left(x_{1}\right)$ degradation & $g /\left(g\right.$ of $\left.x_{2}\right)$ & 42.1 \\
\hline$k_{2}$ & Yield for VFA $\left(x_{3}\right)$ production & $\mathrm{mmol} /\left(g\right.$ of $\left.x_{2}\right)$ & 116.5 \\
\hline$k_{3}$ & Yield for VFA consumption & $\mathrm{mmol} /\left(g\right.$ of $\left.x_{4}\right)$ & 268 \\
\hline$k_{4}$ & Yield for $\mathrm{CO}_{2}$ production & $\mathrm{mmol} / \mathrm{g}$ & 50.6 \\
\hline$k_{5}$ & Yield for $\mathrm{CO}_{2}$ production & $\mathrm{mmol} / \mathrm{g}$ & 343.6 \\
\hline$k_{6}$ & Yield for $\mathrm{ch}_{4}$ production & $\mathrm{mmol} / \mathrm{g}$ & 453 \\
\hline $\bar{\mu}_{1}$ & Maximum acidogenic bacteria $\left(x_{2}\right)$ growth rate & $1 /$ day & 1.25 \\
\hline $\bar{\mu}_{2}$ & Maximum methanogenic bacteria $\left(x_{4}\right)$ growth rate & $1 /$ day & 0.74 \\
\hline$k_{s_{1}}$ & Half saturation constant associated with $x_{1}$ & $g / l$ & 7.1 \\
\hline$k_{s 2}$ & Half saturation constant associated with $x_{3}$ & $\mathrm{mmol} / l$ & 9.28 \\
\hline$k_{i 2}$ & Inhibition constant associated with $x_{3}$ & $\mathrm{mmol} / l$ & 256 \\
\hline$k_{b}$ & Acidity constant of bicarbonate & $\mathrm{mol} / l$ & $6.510^{-7}$ \\
\hline$K_{H}$ & Henry's constant & $\mathrm{mmol} /($ l.atm $)$ & 27 \\
\hline$P_{T}$ & Total preasure & atm & 1.013 \\
\hline$k_{L a}$ & Liquid/gas transfer constant & $1 /$ day & 19.8 \\
\hline
\end{tabular}

to be globally Lipschitz. It is obvious that $B \gamma($.$) can always$ be written under the detailed form:

$$
B \gamma\left(x_{k}\right)=\sum_{i=1}^{m} B_{i} \gamma_{i}(\overbrace{H_{i} x_{k}}^{\vartheta_{i}})
$$

where $H_{i} \in \mathbb{R}^{n_{i} \times n}$ and $B_{i}$ is the $i^{\text {th }}$ column of the matrix $B$.

It is easy to show that the model of anaerobic digestion (1a-1f) can be written under the form (6) with particular parameters that we will provide in a detailed description in Section IV.

\section{B. Some useful preliminaries}

This section is devoted to some preliminaries that will be very useful in the design of the synthesis conditions to ensure the asymptotic convergence of the state observer that we will propose later.

Theorem 3.1 (Mean value theorem [8]): Let $\varphi: \mathbb{R}^{n} \rightarrow$ $\mathbb{R}^{q}$. Let $x, y \in \mathbb{R}^{n}$. We assume that $\varphi$ is differentiable on $C o(x, y)$. Then, there are constant vectors $z_{1}, \ldots, z_{q} \in$ $C o(x, y), z_{i} \neq x, z_{i} \neq y$ for $i=1, \ldots, q$ such that

$$
\varphi(x)-\varphi(y)=\left(\sum_{i, j=1}^{q, n} e_{q}(i) e_{n}^{T}(j) \frac{\partial \varphi_{i}}{\partial x_{j}}\left(z_{i}\right)\right)(x-y)
$$

Lemma 3.1 (a variant of Lipschitz reformulation): Let $\varphi: \mathbb{R}^{n} \rightarrow \mathbb{R}^{q}$ a differentiable function on $\mathbb{R}^{n}$. Then, the following items are equivalent

- $\varphi$ is a globally $\gamma_{\varphi}$-Lipschitz function;

- there exist finite and positive scalar constants $a_{i j}, b_{i j}$ so that for all $x, y \in \mathbb{R}^{n}$ there exist $z_{i} \in \operatorname{Co}(x, y), z_{i} \neq$ $x, z_{i} \neq y$ and functions $\psi_{i j}: \mathbb{R}^{n} \rightarrow \mathbb{R}$ satisfying the following

$$
\varphi(x)-\varphi(y)=\sum_{i, j=1}^{q, n} \psi_{i j}\left(z_{i}\right) \mathcal{H}_{i j}(x-y)
$$

$$
a_{i j} \leq \psi_{i j}\left(z_{i}\right) \leq b_{i j}
$$

where

$$
\psi_{i j}\left(z_{i}\right)=\frac{\partial \varphi_{i}}{\partial x_{j}}\left(z_{i}\right), \mathcal{H}_{i j}=e_{q}(i) e_{n}^{T}(j) .
$$

Notice that this lemma is obvious from the mean value theorem, but it is important to introduce it at this stage, under this formulation, in the aim to simplify the presentation of the proposed observer design method. Indeed, for our technique, we will exploit (9)-(10) instead of a direct use of Lipschitz property.

Lemma 3.2 ([9]): Let $X$ and $Y$ two given matrices of appropriate dimensions. Then, for any symmetric positive definite matrix $S$ of appropriate dimension, the following inequality holds

$$
X^{T} Y+Y^{T} X \leq \frac{1}{2}[X+S Y]^{T} S^{-1}[X+S Y] .
$$

This lemma will be very useful for the main contributions of this paper. It allows providing less restrictive LMI conditions compared to the classical LMI techniques for the considered class of systems.

\section{Observer structure and error dynamics}

In this section, we will propose the structure of the state observer that we will use to estimate the unmeasurable variables of the model (1a-1f). Consider the following general observer structure

$$
\begin{gathered}
\hat{x}_{k+1}=A\left(\rho_{k}\right) \hat{x}_{k}+\sum_{i=1}^{m} B_{i} \gamma_{i}\left(\hat{\vartheta}_{i}\right)+g\left(y_{k}, u_{k}\right) \\
+L\left(\rho_{k}\right)\left(y_{k}-C \hat{x}_{k}\right) \\
\hat{\vartheta}_{i}=H_{i} \hat{x}_{k}+K_{i}\left(\rho_{k}\right)\left(y_{k}-C \hat{x}_{k}\right) \\
L\left(\rho_{k}\right)=L_{0}+\sum_{j=1}^{s} \rho_{k}^{j} L_{j}, \quad K_{i}\left(\rho_{k}\right)=K_{i}^{0}+\sum_{j=1}^{s} \rho_{k}^{j} K_{i}^{j}
\end{gathered}
$$


where $\hat{x}_{k}$ is the estimate of $x_{k}$. The matrices $L_{i} \in \mathbb{R}^{n \times p}$ and $K_{i}^{j} \in \mathbb{R}^{n_{i} \times p}$ are the observer parameters to be determined so that the estimation error $e_{k}=x_{k}-\hat{x}_{k}$ converges asymptotically towards zero.

Since $\gamma($.$) is globally Lipschitz, then from Lemma 3.1$ there exist $z_{i} \in \operatorname{Co}\left(\vartheta_{i}, \hat{\vartheta}_{i}\right)$, functions

$$
\phi_{i j}: \mathbb{R}^{n_{i}} \longrightarrow \mathbb{R}
$$

and constants $a_{i j}, b_{i j}$, such that

$$
B(\gamma(x)-\gamma(\hat{x}))=\sum_{i, j=1}^{m, n_{i}} \phi_{i j}\left(z_{i}\right) \mathcal{H}_{i j}\left(\vartheta_{i}-\hat{\vartheta}_{i}\right)
$$

and

$$
a_{i j} \leq \phi_{i j}\left(z_{i}\right) \leq b_{i j}
$$

where

$$
\phi_{i j}\left(z_{i}\right)=\frac{\partial \gamma_{i}}{\partial \vartheta_{i}^{j}}\left(z_{i}\right), \mathcal{H}_{i j}=B_{i} e_{n_{i}}(j)
$$

For shortness, we set

$$
\phi_{i j} \triangleq \phi_{i j}\left(z_{i}\right) .
$$

Without loss of generality, we assume that $a_{i j}=0$ for all $i=1, \ldots, m$ and $j=1, \ldots, n_{i}$. For more details about this, we refer the reader to [10].

Since $\vartheta_{i}-\hat{\vartheta}_{i}=\left(H_{i}-K_{i}\left(\rho_{k}\right) C\right) e_{k}$, then we have

$$
B\left(\gamma\left(x_{k}\right)-\gamma\left(\hat{x}_{k}\right)\right)=\left[\sum_{i, j=1}^{m, n_{i}} \phi_{i j} \mathcal{H}_{i j}\left(H_{i}-K_{i}\left(\rho_{k}\right) C\right)\right] e_{k}
$$

The dynamics equation of the estimation error is then given by

$$
e_{k+1}=\left(\mathbb{A}_{L}\left(\rho_{k}\right)+\sum_{i, j=1}^{m, n_{i}}\left[\phi_{i j} \mathcal{H}_{i j} \mathbb{H}_{K_{i}}\right]\right) e_{k}
$$

with

$$
\mathbb{A}_{L}=A\left(\rho_{k}\right)-L\left(\rho_{k}\right) C, \mathbb{H}_{K_{i}}=H_{i}-K_{i}\left(\rho_{k}\right) C .
$$

The aim consists in finding the gains $L_{j}$ and $K_{i}^{j}, i=$ $1, \ldots, m, j=1, \ldots, n_{i}$ so that the estimation error (16) turns to be asymptotically stable around zero.

\section{Synthesis conditions and convergence analysis}

The main result related to the convergence analysis of the estimation error is summarized in the following theorem, which provides new LMI conditions.

Theorem 3.2: If there exist symmetric positive definite matrices $\mathbb{P} \in \mathbb{R}^{n \times n}, \mathcal{S}_{i} \in \mathbb{R}^{n_{i} \times n_{i}}$ and matrices $\mathcal{X}_{l} \in \mathbb{R}^{p \times n}$, $\mathcal{Y}_{i}^{l} \in \mathbb{R}^{p \times n_{i}}, i=1, \ldots, m ; l=1, \ldots, s$, of appropriate dimensions so that the following LMI conditions are feasible

$$
\left[\begin{array}{ccc}
\mathbb{M}(\varrho) & {\left[\begin{array}{lll}
\Sigma_{1} & \ldots & \Sigma_{m}
\end{array}\right]} \\
(\star) & & -\Lambda \mathbb{S}
\end{array}\right]<0, \forall \varrho \in \mathbb{V}_{\rho}
$$

with

$$
\begin{gathered}
\mathbb{M}(\varrho)=\left[\begin{array}{ccc}
-\mathbb{P} & \mathbb{M}_{12}(\varrho) & 0 \\
(\star) & -\mathbb{P} & 0 \\
(\star) & 0 & -\mathbb{P}
\end{array}\right] \\
\mathbb{M}_{12}(\varrho)=\left(A_{0}^{T} \mathbb{P}-C^{T} \mathcal{X}_{0}\right)+\sum_{l=1}^{s} \varrho^{l}\left(A_{l}^{T} \mathbb{P}-C^{T} \mathcal{X}_{l}\right) \\
\Sigma_{i}=\left[\Sigma_{i 1}^{T} \ldots \Sigma_{i n_{i}}^{T}\right]^{T}
\end{gathered}
$$

with

$$
\Sigma_{i j}^{T}=\left[\begin{array}{c}
\mathbb{M}_{12}\left(\rho_{k}\right) \mathcal{H}_{i j} \\
0 \\
\mathbb{P} \mathcal{H}_{i j}
\end{array}\right]+\left[\begin{array}{c}
\mathbb{H}\left(\mathcal{S}_{i}, \mathcal{Y}_{i}^{l}\right) \\
0 \\
0
\end{array}\right]
$$

and

$$
\begin{gathered}
\mathbb{H}\left(\mathcal{S}_{i}, \mathcal{Y}_{i, l}\right)=H_{i}^{T} \mathcal{S}_{i}-C^{T}\left(\mathcal{Y}_{i}^{0}+\sum_{l=1}^{s} \varrho^{l} \mathcal{Y}_{i}^{l}\right) \\
\Lambda=\text { block-diag }\left(\Lambda_{1}, \ldots, \Lambda_{m}\right) \\
\Lambda_{i}=\text { block-diag }\left(\frac{2}{b_{i 1}} \mathbb{I}_{n_{i}}, \ldots, \frac{2}{b_{i n_{i}}} \mathbb{I}_{n_{i}}\right) \\
\mathbb{S}=\text { block-diag }\left(\mathbb{S}_{1}, \ldots, \mathbb{S}_{m}\right) \\
\mathbb{S}_{i}=\text { block-diag }(\overbrace{\mathcal{S}_{i}, \ldots, \mathcal{S}_{i}}^{n_{i} \text { times }})
\end{gathered}
$$

then, the estimation error converges asymptotically towards zero. Consequently, the observer parameters $L_{l}$ and $K_{i}^{l}$ are to be computed as follows

$$
L_{l}=\mathbb{P}^{-1} \mathcal{X}_{l}^{T}, \quad K_{i}^{l}=\mathcal{S}_{i}^{-1}\left(\mathcal{Y}_{i}^{l}\right)^{T} .
$$

Proof: We use the following quadratic Lyapunov function to perform the stability analysis of the estimation error

$$
V\left(e_{k}\right)=e_{k}^{T} \mathbb{P} e_{k}, \quad \mathbb{P}=\mathbb{P}^{T}>0 .
$$

By calculating $\Delta V=V\left(e_{k+1}\right)-V\left(e_{k}\right)$ along the trajectories of (16), we obtain

$$
\begin{aligned}
\Delta V= & e_{k}^{T}\left[\left(\mathbb{A}_{L}\left(\rho_{k}\right)+\sum_{i, j=1}^{m, n_{i}}\left[\phi_{i j} \mathcal{H}_{i j} \mathbb{H}_{K_{i}}\right]\right)^{T} \mathbb{P}\right. \\
& \left.\times\left(\mathbb{A}_{L}\left(\rho_{k}\right)+\sum_{i, j=1}^{m, n_{i}}\left[\phi_{i j} \mathcal{H}_{i j} \mathbb{H}_{K_{i}}\right]\right)-\mathbb{P}\right] e
\end{aligned}
$$

Hence, $\Delta V<0$ if the following inequality holds

$$
\begin{aligned}
& \left(\mathbb{A}_{L}\left(\rho_{k}\right)+\sum_{i, j=1}^{m, n_{i}}\left[\phi_{i j} \mathcal{H}_{i j} \mathbb{H}_{K_{i}}\right]\right)^{T} \mathbb{P} \\
& \left.\quad \times\left(\mathbb{A}_{L}\left(\rho_{k}\right)+\sum_{i, j=1}^{m, n_{i}}\left[\phi_{i j} \mathcal{H}_{i j} \mathbb{H}_{K_{i}}\right]\right)-\mathbb{P}\right]<0 .
\end{aligned}
$$


Inequality (29) is equivalent, by Schur lemma, to

$$
\left[\begin{array}{ccc}
-\mathbb{P}+\Sigma_{11}+\Sigma_{11}^{T} & \mathbb{A}_{L}^{T}\left(\rho_{k}\right) \mathbb{P} & \Sigma_{13}^{T} \\
(\star) & -\mathbb{P} & 0 \\
(\star) & 0 & -\mathbb{P}
\end{array}\right]<0
$$

where

$$
\begin{gathered}
\Sigma_{11}=\sum_{i, j=1}^{m, n_{i}}\left(\phi_{i j} \mathbb{A}_{L}^{T}\left(\rho_{k}\right) \mathbb{P} \mathcal{H}_{i j} \mathbb{H}_{K_{i}}\right), \\
\Sigma_{13}=\sum_{i, j=1}^{m, n_{i}}\left(\phi_{i j} \mathbb{P}_{i j} \mathbb{H}_{K_{i}}\right) .
\end{gathered}
$$

On the other hand, inequality (30) can be rewritten under the form

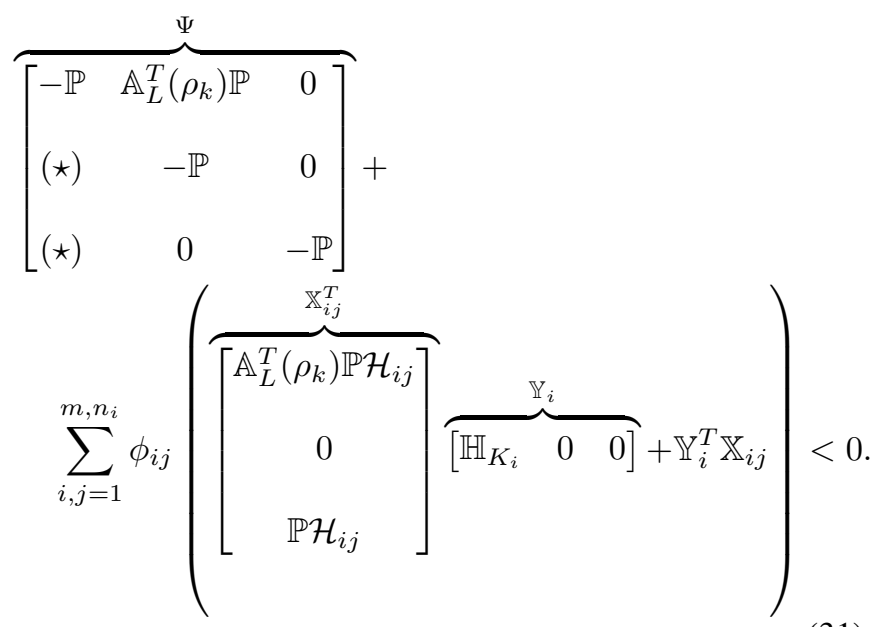

Now, by applying Lemma 3.2 we have

$$
\mathbb{X}_{i j}^{T} \mathbb{Y}_{i}+\mathbb{Y}_{i}^{T} \mathbb{X}_{i j} \leq \frac{1}{2}\left(\mathbb{X}_{i j}+\mathbb{S}_{i j} \mathbb{Y}_{i}\right)^{T} \mathbb{S}_{i j}^{-1} \overbrace{\left(\mathbb{X}_{i j}+\mathbb{S}_{i j} \mathbb{Y}_{i}\right)}^{\Sigma_{i j}}
$$

for any symmetric positive definite matrices $\mathbb{S}_{i j}$. Since the matrix block $\mathbb{Y}_{i}$ does not depend on the index $j$ and depends on the same $K_{i}\left(\rho_{k}\right)$, then to obtain an LMI we need to put

$$
\mathbb{S}_{i j}=\mathcal{S}_{i}, \forall(i, j)
$$

with $\mathcal{S}_{i} \in \mathbb{R}^{n_{i} \times n_{i}}$.

Consequently, from (14) and the fact that $a_{i j}=0$, inequality (31) holds if

$$
\Psi-\sum_{i, j=1}^{m, n_{i}}\left(\Sigma_{i j}^{T}\left(-\frac{2}{b_{i j}} \mathbb{S}_{i j}\right)^{-1} \Sigma_{i j}\right)<0 .
$$

Using Schur lemma, inequality (32) is equivalent to

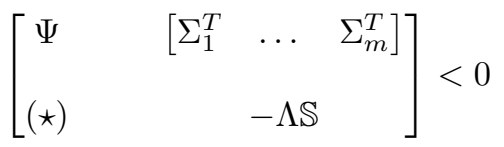

Finally, we use the change of variables $\mathcal{X}_{i}=L_{i}^{T} \mathbb{P}$ and $\mathcal{Y}_{i}^{l}=\left(K_{i}^{l}\right)^{T} \mathcal{S}_{i}$, and since (33) is affine in $\rho_{k}$, then the convexity principle [11] leads to (18). This ends the proof.

\section{Simulation Results: Application of the} Proposed OBSERVER ON THE AD MODEL

The objective of this paper is to estimate the main state variables of the AD model (1a-1f). Therefore, to apply the designed observer in Section III, we first write the model (1a1f) in the form (6). This is done by using the first order Euler discretization method with sampling time $T_{s}$. After discretization, the model (1a-1f) is written under the form (6) with the following parameters

$$
\begin{aligned}
& \rho_{k}=u_{\text {out }}(k), \quad A_{0}=\mathbb{I}_{6} \\
& A_{1}=-T_{s} \times \text { block-diag }(1, \alpha, 1, \alpha, 1,1) \\
& \gamma\left(x_{k}\right)=\left[\begin{array}{c}
\mu_{1}\left(x_{1 k}\right) x_{2 k} \\
\mu_{2}\left(x_{3 k}\right) x_{4 k}
\end{array}\right] \\
& B=T_{s}\left[\begin{array}{cccccc}
-k_{1} & 1 & k_{2} & 0 & k_{4} & 0 \\
0 & 0 & -k_{3} & 1 & k_{5} & 0
\end{array}\right]^{T} \\
& g\left(y_{k}, u_{k}\right)=\left[\begin{array}{c}
u_{1}(k) S_{1 i n} \\
0 \\
u_{1}(k)\left(S_{2 i n}+S_{2 a d}(k)\right) \\
0 \\
u_{1}(k) C_{i n}-q_{c}(k) \\
u_{1}(k) Z_{i n}+u_{2}(k) Z_{a d}
\end{array}\right] \\
& C=\left[\begin{array}{llllll}
1 & 0 & 0 & 0 & 0 & 0 \\
0 & 0 & 1 & 0 & 0 & 0 \\
0 & 0 & 0 & 0 & 0 & 1
\end{array}\right]
\end{aligned}
$$

Moreover, for the observer design we have, $m=2, s=1$, $n_{i}=2, \gamma_{1}\left(x_{k}\right)=\mu_{1}\left(x_{1 k}\right) x_{2 k}, \gamma_{2}\left(x_{k}\right)=\mu_{2}\left(x_{3 k}\right) x_{4 k}$,

$$
\begin{aligned}
& H_{1}=\left[\begin{array}{llllll}
1 & 0 & 0 & 0 & 0 & 0 \\
0 & 1 & 0 & 0 & 0 & 0
\end{array}\right] \\
& H_{2}=\left[\begin{array}{llllll}
0 & 0 & 1 & 0 & 0 & 0 \\
0 & 0 & 0 & 1 & 0 & 0
\end{array}\right] \\
& B_{1}=T_{s}\left[\begin{array}{llllll}
-k_{1} & 1 & k_{2} & 0 & k_{4} & 0
\end{array}\right]^{T} \\
& B_{2}=T_{s}\left[\begin{array}{llllll}
0 & 0 & -k_{3} & 1 & k_{5} & 0
\end{array}\right]^{T}
\end{aligned}
$$

The simulations have been run for $T_{s}=0.001$ (day), $\rho_{\min }=0, \rho_{\max }=0.62(1 /$ day $), Z_{a d}=700$ $(\mathrm{mmol} / \mathrm{l}), S_{2 a d}=0, S_{1 i n}=16(\mathrm{~g} / \mathrm{l}), S_{2 i n}=170$ $(\mathrm{mmol} / l), C_{i n}=76.15(\mathrm{mmol} / l), Z_{\text {in }}=200(\mathrm{mmol} / l)$ and the parameter values given in Table I. Moreover, the system and the observer were initialized, respectively, at $x(0)=[2,0.5,12,0.7,53.48,55]^{T}$ and $\hat{x}(0)=$ $[2,1,12,0.4,58.48,55]^{T}$. The simulation results are depicted in Figures 2-4, they were obtained after solving the LMIs (18), which were found feasible by using the LMI toolbox of MATLAB. These LMIs provided the observer gains which ensure the asymptotic convergence of the estimation error to zero. Indeed, as it can be seen from Figures 24, although the large initial estimation errors, the observer 
states are converging very well to the simulated system state variables. Moreover, we want to say that the convergence of the methanogenic bacteria concentration, which plays a key role in supervision and monitoring of AD processes, is relatively fast.

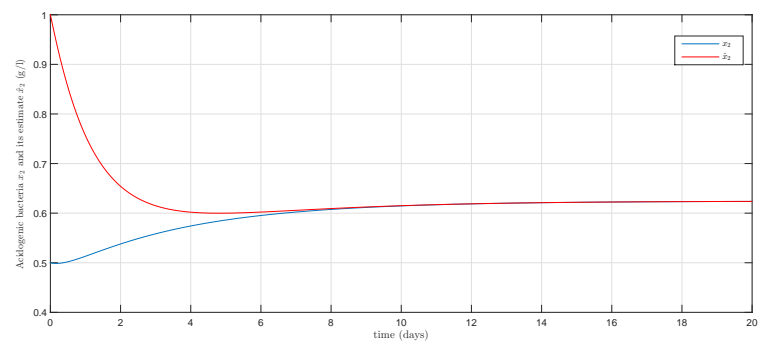

Fig. 2. Acidogenic bacteria $x_{2}$ and its estimate $\hat{x}_{2}(g / l)$.

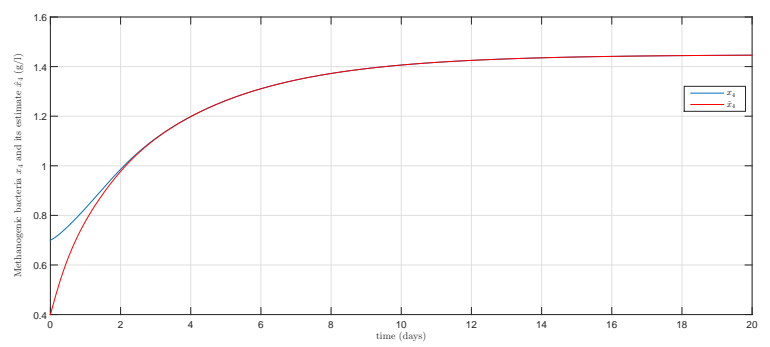

Fig. 3. Mathenogenic bacteria $x_{4}$ and its estimate $\hat{x}_{4}(g / l)$.

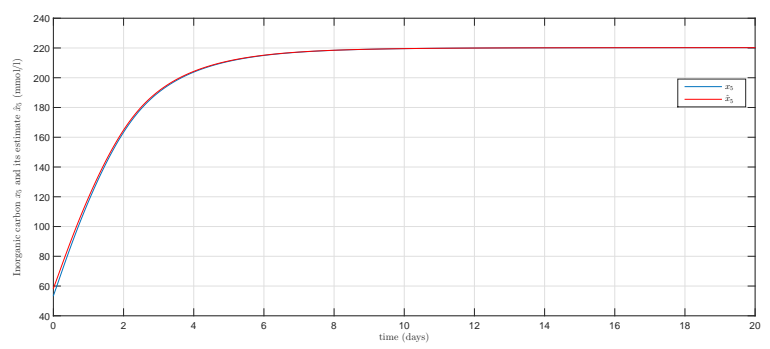

Fig. 4. Inorganic Carbon $x_{5}$ and its estimate $\hat{x}_{5}(\mathrm{mmol} / \mathrm{l})$.

\section{Conclusions}

In the present paper, we have first modified an anaerobic digestion model to adapt it for further research objectives. Then, we have proposed a suitable nonlinear observer to estimate the key state variables of the proposed model. The designed observer is based on new and less restrictive LMI conditions. Actually, this was possible due to the exploit of the new judicious reformulation of Young's inequality.

The simulation results being promising, we target in the near future to extend the methodology for systems with unknown inputs and evaluate the observer robustness when the system is disturbed and the measurements are corrupted. Further, we also plan to investigate the optimal control problem of biogas plants based on the designed nonlinear observer.

\section{REFERENCES}

[1] G. Bastin and D. Dochain, On-line Estimation and Adaptive Control of Bioreactors, ser. Process Measurement and Control. Elsevier, 1990.

[2] O. Bernard and J. Gouzé, "Closed loop observers bundle for uncertain biotechnological models," Journal of Process Control, vol. 14, no. 7, pp. 765-774, 2004.

[3] J. P. Gauthier, H. Hammouri, and S. Othman, "A simple observer for nonlinear systems, applications to bioreactors," IEEE Transactions on Automatic Control, vol. 37, no. 6, pp. 875-880, 1992.

[4] C. Kravaris, J. Hahn, and Y. Chu, "Advances and selected recent ddevelopments in state and parameter estimation," Computers and Chemical Engineering, vol. 51, pp. 111 - 123, 2013.

[5] A. Zemouche, R. Rajamani, B. Boulkroune, H. Rafaralahy, and M. Zasadzinski, "Convex optimization based dual gain observer design for Lipschitz nonlinear systems," in IEEE American Control Conference, Boston, MA, USA., July 2016.

[6] M. D. K. Chaib Draa, H. Voos and M.Alma, "A formal modeling framework for anaerobic digestion systems," in Proceedings of the $201517^{\text {th }}$ UKSIM-AMSS International Conference on Modelling and Simulation. IEEE Computer Society, 2015, pp. 426-431.

[7] D. D. A. G. O. Bernard, Z. Hadj-Sadok and J. Steyer, "Dynamical model development and parameter identification for an anaerobic wastewater treatment process," Biotechnology and Bioengineering, vol. 75, no. 4, pp. $424-438,2001$.

[8] A. Zemouche, M. Boutayeb, and G. I. Bara, "Observers for a class of Lipschitz systems with extension to $\mathcal{H}_{\infty}$ performance analysis," Systems \& Control Letters, vol. 57, no. 1, pp. 18-27, 2008.

[9] A. Zemouche, R. Rajamani, B. Boulkroune, H. Rafaralahy, and M. Zasadzinski, " $\mathcal{H}_{\infty}$ circle criterion observer design for Lipschitz nonlinear systems with enhanced LMI conditions," in IEEE American Control Conference, Boston, MA, USA., July 2016.

[10] M. Arcak and P. Kokotovic, "Observer-based control of systems with slope-restricted nonlinearities," IEEE Transactions on Automatic Control, vol. 46, no. 7, pp. 1146-1150, 2001.

[11] S. Boyd, L. El Ghaoui, E. Feron, and V. Balakrishnan, "Linear matrix inequalities in system and control theory," in SIAM Studies in Applied Mathematics, Philadelphia, USA, 1994. 